\title{
A Modified Strip-Yield-Saturation-Induction Model Solution for Cracked Piezoelectromagnetic Plate
}

\author{
R. R. Bhargava and Pooja Raj Verma \\ Department of Mathematics, Indian Institute of Technology Roorkee, Roorkee 247667, India \\ Correspondence should be addressed to Pooja Raj Verma; poojarajvs@gmail.com
}

Received 9 May 2014; Accepted 2 July 2014; Published 14 August 2014

Academic Editor: Song Cen

Copyright (C) 2014 R. R. Bhargava and P. R. Verma. This is an open access article distributed under the Creative Commons Attribution License, which permits unrestricted use, distribution, and reproduction in any medium, provided the original work is properly cited.

\begin{abstract}
A strip-yield-saturation-induction model is proposed for an impermeable crack embedded in piezoelectromagnetic plate. The developed slide-yield, saturation, and induction zones are arrested by distributing, respectively, mechanical, electrical, and magnetic loads over their rims. Two cases are considered: when saturation zone exceeds induction zone and vice-versa. It is assumed that developed slide-yield zone is the smallest because of the brittle nature of piezoelectromagnetic material. Fourier integral transform technique is employed to obtain the solution. Closed form analytic expressions are derived for developed zones lengths, crack sliding displacement, crack opening potential drop, crack opening induction drop, and energy release rate. Case study presented for $\mathrm{BaTiO}_{3}-\mathrm{CoFe}_{2} \mathrm{O}_{4}$ shows that crack arrest is possible under small-scale mechanical, electrical, and magnetic yielding.
\end{abstract}

\section{Introduction}

The work on magnetoelectroelastic (MEE) fracture problem was started late back in the last century. The field is a natural extension of piezoelectric media since electricity and magnetism go in hand. Due to coupling effect of magneto-, electro-, and elastic fields, MEE materials become more popular than piezoelectric materials and serve as the excellent sensor, actuator, and transducer.

Wang and Shen [1] obtained energy release rate for a mode-III magnetoelectroelastic media based on the concept of energy-momentum tensor. Based on the extended Stroh formalism combined with complex variable technique, Green's function is obtained for an infinite two-dimensional anisotropic MEE media containing an elliptic cavity which degenerates into a slit crack, by Jinxi et al. [2]. Sih and Song [3] proposed a model which showed that crack growth in a magnetoelectroelastic material could be suppressed by increasing the magnitude of piezomagnetic constants in relation to these for piezoelectricity. They [4] further derived energy density function for cracked MEE medium and studied the additional magnetic strictive effect which could influence crack initiation as applied field direction is altered. Wang and Mai [5] addressed the problem of a crack in a MEE medium possessing coupled piezoelectric, piezomagnetic, and magnetoelastic effects. Wang and Mai [6] further extended above problem to calculate a conservative integral based on governing equations for MEE media. Gao et al. [7] investigated the fracture mechanics for an elliptic cavity in a MEE solid under remotely applied uniform in-plane electromagnetic and/or antiplane mechanical loadings. Reducing cavity into a crack they considered two extreme cases for impermeable crack and permeable crack cases. $\mathrm{Hu}$ and $\mathrm{Li}$ [8] obtained singular stress, electric and magnetic fields in MEE strip containing a Griffith crack under longitudinal shear for a crack situated symmetrically and oriented in a direction parallel to the edges of the strip. Tian and Rajapakse [9] obtained the solution for single, multiple, and slowly growing impermeable cracks in a MEE solid using generalized edge dislocation theory. The solution for an elliptic cavity in an infinite two-dimensional MEE medium subjected to remotely applied uniform combined mechanical, electric, and magnetic loadings under permeable crack face boundary condition along the cavity of the surface had been obtained by Zhao et al. [10]. Wang and Mai [11] discussed different electromagnetic boundary conditions on permeable and impermeable crack faces in 
a MEE material. Ma et al. [12] addressed an antiplane problem of a functionally graded MEE strip containing an internal or edge permeable/impermeable crack lying transversely to the edges of the strip. A mixed boundary value problem was solved by Zhong and Li [13] for a crack in MEE solid. Kirilyuk [14] gave a method which enabled to find stress intensity factor (SIF) for a cracked MEE body directly from the analogous problem of elasticity. Zhao and Fan [15] proposed a strip electric-magnetic breakdown model for an electrically and magnetically impermeable crack in MEE media using extended Stroh formalism and the extended dislocation modelling of a crack. They have also proposed [16] a strip electric-magnetic polarization saturation model for an infinite and finite MEE strip. Using finite element method Krahulec et al. [17] discussed a crack problem for MEE solid under various electromagnetic boundary conditions on the crack rims. Recently, we, Bhargava and Verma [18], proposed a mechanical, electrical, and induction yield model (based on Dugdale [19] type model) for a cracked MEE 2D media.

In present paper, we have generalized the classical Dugdale model [19] and propose strip-yield-saturation-induction yield model for an unbounded cracked piezoelectromagnetic plate with electric and magnetic polarization in $z$ direction. Due to mechanical brittleness, it is assumed that developed mechanical yielding zone is the smallest zone. In the problem, two cases are considered: Case I is when developed saturation zone is bigger than induction zone and Case II is when developed saturation zone is smaller than induction zone. Fourier transform technique is employed to obtain the solution and derived closed form expressions for developed zone sizes, energy release rate, crack opening potential drop, and crack opening induction drop. To test the proposed model, a case study is presented for $\mathrm{BaTiO}_{3}-$ $\mathrm{CoFe}_{2} \mathrm{O}_{4}$ piezoelectromagnetic ceramic. Numerical results are presented graphically and these confirm that the proposed model is capable of crack arrest under small-scale electrical and magnetic yielding.

\section{Fundamental Formulation and Solution Methodology}

As are well known, the out-of-plane displacement and inplane electric and magnetic fields problems may be expressed as

$$
\begin{gathered}
u_{x}=0, \quad u_{y}=0, \quad u_{z}=w(x, y), \\
E_{x}=E_{x}(x, y), \quad E_{y}=E_{y}(x, y), \quad E_{z}=0, \\
H_{x}=H_{x}(x, y), \quad H_{y}=H_{y}(x, y), \quad H_{z}=0,
\end{gathered}
$$

in $x y z$-orthogonal coordinate system.

For transversely isotropic magnetoelectroelastic medium which is poled along $z$-direction, the constitutive equations for stress, $\tau_{i z}$, electric-displacement, $D_{i}$, and magnetic induction, $B_{i}(i=x, y)$, components may be expressed as

$$
\begin{aligned}
& \tau_{i z}=c_{44} w_{, i}+e_{15} \phi_{, i}+h_{15} \psi_{, i}, \\
& D_{i}=e_{15} w_{, i}-\varepsilon_{11} \phi_{, i}-\beta_{11} \psi_{, i},
\end{aligned}
$$

$$
B_{i}=h_{15} w_{, i}-\beta_{11} \phi_{, i}-\gamma_{11} \psi_{, i}
$$

where $c_{44}, e_{15}, h_{15}, \varepsilon_{11}, \beta_{11}$, and $\gamma_{11}$ denote elastic constant, piezoelectric coefficient, piezomagnetic coefficient, magnetic permeability, and dielectric coefficient, respectively. A comma in subscript denotes the partial differentiation with respect to argument following it.

The equilibrium equations in the absence of body force, electric charge, and magnetic flux, respectively, are expressed as

$$
\tau_{i j, i}=0, \quad D_{i, i}=0, \quad B_{i, i}=0 .
$$

The gradient equations for strain components, $\gamma_{i j}$, magnetic field, $H_{i}$, and electric field, $E_{i}$, may be written as

$$
\gamma_{i z}=w_{, i}, \quad E_{i}=-\phi_{, i}, \quad H_{i}=-\psi_{, i},
$$

where $i, j=x, y$.

And the governing equations for electrically and magnetically poled piezoelectromagnetic ceramic under modeIII deformation may be written as

$$
\left(\begin{array}{ccc}
c_{44} & e_{15} & h_{15} \\
e_{15} & -\varepsilon_{11} & -\beta_{11} \\
h_{15} & -\beta_{11} & -\gamma_{11}
\end{array}\right) \nabla^{2} g=0
$$

where $\nabla^{2}=\left(\partial^{2} / \partial x^{2}\right)+\left(\partial^{2} / \partial y^{2}\right)$ is two-dimensional Laplacian operator and $g=[w, \phi, \psi]^{T}$. The superscript $T$ denotes the transpose of matrix.

To obtain the desired potentials $w, \phi$, and $\psi$, equation (5) is solved by taking Fourier integral transform. The solution of corresponding ordinary differential equation is given by

$$
\begin{aligned}
& \tilde{\mathfrak{g}}^{+}= \begin{cases}C e^{s y}, & s<0, \\
F e^{-s y}, & s>0,\end{cases} \\
& \tilde{\mathfrak{g}}^{-}= \begin{cases}C e^{s y}, & s>0, \\
F e^{-s y}, & s<0,\end{cases}
\end{aligned}
$$

where $\sim$ over the functions denotes its Fourier transform and superscripts + and - denote the value of function on the rims of crack as approached from $y>0$ and $y<0$ planes, respectively. $C$ and $F$ are $3 \times 1$ column vectors and $s$ is Fourier transform variable.

For convenience of calculations, the boundary conditions of the problem are also written in terms of their Fourier transform. Fourier transform of constitutive equations (2) may be written as

$$
\tilde{\mathbf{t}}(s, q)=-i s B \frac{\partial \widetilde{g}(s, q)}{\partial q}=-i B \frac{\partial \widetilde{g}(s, y)}{\partial y}
$$

where $\mathbf{t}=\left[\tau_{y z}, D_{y}, B_{y}\right]^{T} ; q=-i s y$ and

$$
B=i\left(\begin{array}{ccc}
c_{44} & e_{15} & h_{15} \\
e_{15} & -\varepsilon_{11} & -\beta_{11} \\
h_{15} & -\beta_{11} & -\gamma_{11}
\end{array}\right) .
$$


Solution of equation (8) may be given as

$$
\begin{aligned}
& \tilde{\mathbf{t}}^{+}(s, y)= \begin{cases}i s B C e^{s y}, & s<0, \\
-i s B F e^{-s y}, & s>0,\end{cases} \\
& \tilde{\mathbf{t}}^{-}(s, y)= \begin{cases}i s B C e^{s y}, & s>0, \\
-i s B F e^{-s y}, & s<0 .\end{cases}
\end{aligned}
$$

The continuity condition for traction on $y=0$ and $|x|<a$ yields

$$
-\widetilde{\mathbf{t}}^{+}(s, 0)=k \widetilde{g}^{+}(s, 0) ; \quad \widetilde{\mathbf{t}}^{-}(s, 0)=k \widetilde{g}^{-}(s, 0) .
$$

Equations (10) and (11) together yield

$$
k \widetilde{g}^{+}(s, 0)+k \widetilde{g}^{-}(s, 0)=0,
$$

where $k=\left\{\begin{array}{l}\text { is } B, s<0 \\ -i s B, s>0 .\end{array}\right.$

The jump in displacement, electric-displacement, and magnetic induction are defined by

$$
\Delta g(x)=g^{+}(x, 0)-g^{-}(x, 0) .
$$

And the dislocation function, $f_{1}(x)$, dipole density function, $f_{2}(x)$, and induction density function, $f_{3}(x)$, are defined as

$$
\mathbf{f}(\mathbf{x})=\left[f_{1}(x), f_{2}(x), f_{3}(x)\right]^{T}=\frac{d}{d x} \Delta g(x)
$$

Taking Fourier transform of (14) one obtains

$$
\Delta \tilde{g}(x)=\frac{i}{s} \int_{-a}^{a} \mathbf{f}(\mathbf{x}) e^{i s x} d x .
$$

Equations (12) and (15) may also be expressed as

$$
\widetilde{\mathbf{t}}(s, 0)=-\frac{1}{2} k \Delta \tilde{g}(x) .
$$

Inverse Fourier transform of the above equation and (11) and (16) yields a system of integral equations to determine $\mathbf{f}(\mathbf{x})$ as

$$
-\frac{B}{\pi} \int_{-a}^{a} \frac{\mathbf{f}(\mathbf{t})}{t-x} d t=\mathbf{t}(x), \quad|x|<\infty, y=0 .
$$

\section{The Problem}

A piezoelectromagnetic ceramic plate occupies entire $x y$ plane and is thick enough in $z$-direction to allow antiplane deformations. The plate is poled along positive $z$-direction both magnetically and electrically. The plate is cut along a hairline, quasi-stationary straight crack. The crack occupies the interval $[-a, a]$ on $x$-axis.

Remote boundary of the plate is prescribed:

(i) an antiplane shear stress: $\tau_{y z}=\tau_{\infty}$,

(ii) in-plane:
(a) electric displacement: $D_{y}=D_{\infty}$,
(b) magnetic induction: $B_{y}=B_{\infty}$.

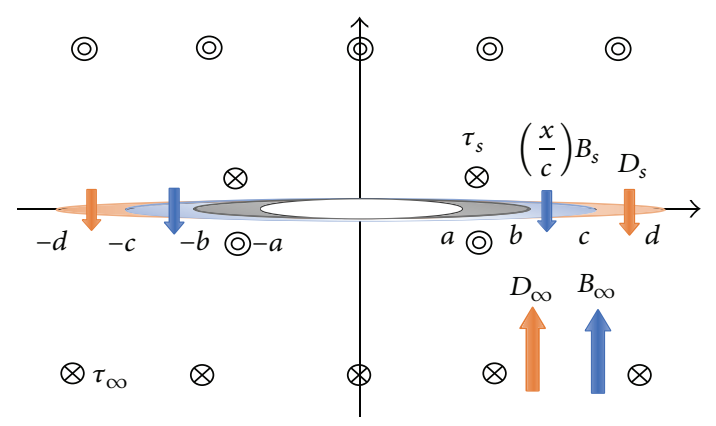

FIGURE 1: The schematic representation of the problem for Case I.

Consequently, the crack rims open in self-similar fashion. Hence a strip-yield zone, a strip-saturation zone, and a stripinduction zone protrude ahead of each tip of the crack. These strip zones are assumed to occupy the interval $a \leq|x| \leq b$, $a \leq|x| \leq d$ and $a \leq|x| \leq c$ on $x$-axis, respectively. Two cases are investigated.

Case I. When developed saturation zone exceeds developed induction zone, $(c<d)$.

To arrest the crack from further opening, the rims of saturation zone are subjected to normal cohesive in-plane saturation limit electrical displacement; $D_{y}=D_{s}$. And the rims of developed induction zone are subjected to in-plane normal cohesive magnetic load; $B_{y}=(x / c) B_{s}$, where $B_{s}$ denotes saturation limit of magnetic induction and $x$ is any point on induction zone.

Case II. When developed saturation zone is smaller than developed induction zone, $(c>d)$.

For this case, developed induction zone rims are prescribed in-plane, normal cohesive saturation limit of magnetic induction, $B_{y}=B_{s}$, and developed saturation zone is subjected to in-plane normal cohesively linearly varying saturation limit electric displacement, $D_{y}=(x / d) D_{s}$, where $D_{s}$ is saturation limit electrical displacement and $x$ is any point on saturation zone.

For both considered cases, developed slide-yield zone is taken to be the smallest $(b<c \& d)$ and is prescribed cohesive yield-point shear stress, $\tau_{y z}=\tau_{s}$.

\section{Case I: Solution and Applications}

Schematically, the configuration of the problem for Case I is depicted in Figure 1.

The traction conditions $t_{i}^{\mathrm{I}}(i=1,2,3)$ denote prescribed mechanical, electric, and magnetic loads, respectively, and for this case given as

(i) $t_{1}^{\mathrm{I}}= \begin{cases}-\tau_{\infty}, & |x| \leq a, \\ -\tau_{\infty}+\tau_{s}, & a<|x| \leq b,\end{cases}$

(ii) $t_{2}^{\mathrm{I}}= \begin{cases}-D_{\infty}, & |x| \leq a, \\ -D_{\infty}+D_{s}, & a<|x| \leq d,\end{cases}$ 


$$
\text { (iii) } t_{3}^{\mathrm{I}}= \begin{cases}-B_{\infty} & |x| \leq a, \\ -B_{\infty}+\left(\frac{x}{c}\right) B_{s} & a<|x| \leq c,\end{cases}
$$

where superscript I denotes that the quantity refers to Case I.

The boundary conditions (i) to (iii) together with traction continuity condition on $x$-axis yield $f_{1}(x)=0$ for $|x|>b$, $f_{2}(x)=0$ for $|x|>d$, and $f_{3}(x)=0$ for $|x|>c$. (17) as

Consequently, the solution for $f_{1}(x)$ may be written using

$$
\int_{-b}^{b} \frac{f_{1}(t)}{t-x} d t=\pi G_{1 j} t_{j}^{\mathrm{I}}(x),
$$

provided that

$$
\int_{-b}^{b} \frac{G_{1 j} t_{j}^{\mathrm{I}}}{\sqrt{b^{2}-t^{2}}} d t=0
$$

which on evaluation leads to a transcendental equation to determine $b$ :

$$
\begin{gathered}
2\left(G_{11} \tau_{s}+G_{12} D_{s}\right) \cos ^{-1}\left(\frac{a}{b}\right)+\left\{\frac{\left(2 B_{s} G_{13}\right)}{c}\right\} \sqrt{b^{2}-a^{2}} \\
=\pi\left(G_{11} \tau_{\infty}+G_{12} D_{\infty}+G_{13} B_{\infty}\right) .
\end{gathered}
$$

Hence the slide-yield zone is determined using $|b-a|$.

Using (21), the solution of (19) may be written as

$$
\begin{aligned}
\pi f_{1}(x)= & \left(G_{11} \tau_{s}+G_{12} D_{s}+\left(\frac{x}{c}\right) G_{13} B_{s}\right) \\
& \times[\omega(x, a, b)-\omega(-x, a, b)] \\
& +\left\{\frac{\left(2 B_{s} G_{13}\right)}{c}\right\} \sqrt{b^{2}-x^{2}} \cos ^{-1}\left(\frac{a}{b}\right),
\end{aligned}
$$

where $\omega(x, a, b)=\cosh ^{-1}\left|\left(b^{2}-a x\right) / b(x-a)\right|$.

Analogously, the solution for $f_{3}(x)$ is written using equation (16) under the constrain

$$
\begin{aligned}
& G_{31}\left(\tau_{\infty}-\tau_{s}\right) \\
& \times \cos ^{-1}\left(\frac{b}{c}\right)+B_{s} G_{33} \sqrt{1-\left(\frac{a}{c}\right)^{2}}+\left(G_{32} D_{s}+G_{31} \tau_{s}\right) \\
& \quad \times \cos ^{-1}\left(\frac{a}{c}\right) \\
& =\left(\frac{\pi}{2}\right)\left[G_{31} \tau_{\infty}+G_{32} D_{\infty}+G_{33} B_{\infty}\right]
\end{aligned}
$$

as

$$
\begin{aligned}
\pi f_{3}(x)= & G_{31}\left(\tau_{\infty}-\tau_{s}\right)[\omega(x, b, c)-\omega(-x, b, c)] \\
& +2 B_{s} G_{33} \sqrt{1-\left(\frac{x}{c}\right)^{2} \cos ^{-1}\left(\frac{a}{c}\right)} \\
& +\left(G_{31} \tau_{s}+G_{32} D_{s}+\left(\frac{x}{c}\right) G_{33} B_{s}\right) \\
& \times[\omega(x, a, c)-\omega(-x, a, c)] .
\end{aligned}
$$

Equation (23) determines $c$ for known value of $b$ which is obtained using (21).

The dipole density function, $f_{2}(x)$, is determined from

$$
\int_{-d}^{d} \frac{H_{2 j} f_{j}(t)}{t-x} d t=\pi t_{2}^{I}(x),
$$

where $j=1,2,3$ and $G=H^{-1}$ with

$$
H=\left(\begin{array}{ccc}
c_{44} & e_{15} & h_{15} \\
e_{15} & -\varepsilon_{11} & -\beta_{11} \\
h_{15} & -\beta_{11} & -\gamma_{11}
\end{array}\right) .
$$
dition

The solution of equation (25) is obtained under the con-

$$
\frac{d}{a}=\sec \left(\frac{\pi}{2} \frac{D_{\infty}}{D_{s}}\right)
$$

as

$$
\begin{aligned}
H_{22} f_{2}(x)= & -H_{21} f_{1}(x)-H_{23} f_{3}(x) \\
& +\left(\frac{D_{s}}{\pi}\right)[\omega(x, a, d)-\omega(-x, a, d)] .
\end{aligned}
$$

Equation (27) enables to determine $d$. The saturation zone length is then determined using $|d-a|$.

\subsection{Applications for Case I}

4.1.1. Crack Sliding Displacement (CSD), Crack Opening Induction Drop (COI), and Crack Opening Potential Drop (COP). Quantities of interest, namely, CSD, COI, and COP, are obtained in closed form analytic expressions in this section.

The crack sliding displacement, $\Delta^{\mathrm{I}} w(x)$, is obtained using the following formula:

$$
\Delta^{\mathrm{I}} w(x)=-\int_{-b}^{x} f_{1}(x) d x, \quad-b \leq x \leq b .
$$

Substituting $f_{1}(x)$ from (22) and evaluating, one obtains

$$
\begin{aligned}
\Delta^{\mathrm{I}} w(x) & \\
=\left(\frac{1}{\pi}\right)[ & \left(G_{11} \tau_{s}+G_{12} D_{s}\right) \\
& \times[(x-a) \omega(x, a, b)+(x+b) \omega(-x, a, b)] \\
& -\left(\frac{\left(B_{s} G_{13}\right)}{c}\right)
\end{aligned}
$$




$$
\begin{aligned}
& \times\left[\left(x^{2}-a^{2}\right)\{\omega(x, a, b)+\omega(-x, a, b)\}\right. \\
& \left.-2 a \sqrt{b^{2}-a^{2}}\left(\cos ^{-1}\left(\frac{a}{b}\right)-\pi\right)\right] \\
& -\left(\frac{\left(B_{s} G_{13}\right)}{c}\right) \cos ^{-1}\left(\frac{a}{b}\right) \\
& \left.\times\left[x \sqrt{b^{2}-x^{2}}+b^{2} \sin ^{-1}\left(\frac{x}{b}\right)-\frac{\left(b^{2} \pi\right)}{2}\right]\right] .
\end{aligned}
$$

Crack opening induction drop (COI), $\Delta^{\mathrm{I}} \psi(x)$, across the developed induction zone rims is calculated using the definition

$$
\begin{aligned}
\Delta^{\mathrm{I}} \psi(x)= & -\int_{-c}^{x} f_{3}(x) d x, \quad-c \leq x \leq c, \\
= & \left(\frac{1}{\pi}\right) G_{31}\left(\tau_{\infty}-\tau_{s}\right) \\
& \times[(x-b) \omega(x, b, c)+(x+b) \omega(-x, b, c)] \\
& -\left(\frac{\left(B_{s} G_{33}\right)}{(\pi c)}\right) \cos ^{-1}\left(\frac{a}{c}\right) \\
& \times\left[x \sqrt{c^{2}-x^{2}}+c^{2} \sin ^{-1}\left(\frac{x}{c}\right)-\frac{\left(c^{2} \pi\right)}{2}\right] \\
& +\left(\frac{\left(G_{31} \tau_{s}+G_{32} D_{s}\right)}{\pi}\right) \\
& \times[(x-a) \omega(x, a, c)+(x+a) \omega(-x, a, c)] \\
& -\left(\frac{\left(B_{s} G_{13}\right)}{(c \pi)}\right) \\
& \times\left[\left(x^{2}-a^{2}\right)\{\omega(x, a, c)+\omega(-x, a, c)\}\right. \\
& \left.-(2 a) \sqrt{c^{2}-a^{2}}\left(\cos ^{-1}\left(\frac{a}{c}\right)-\pi\right)\right] .
\end{aligned}
$$

And the crack opening potential drop (COP), $\Delta^{\mathrm{I}} \phi(x)$, is calculated using

$$
\begin{aligned}
\Delta^{\mathrm{I}} \phi(x)= & -\int_{-d}^{x} f_{2}(x) d x, \quad-d \leq x \leq d, \\
= & -\left(\frac{H_{21}}{H_{22}}\right) \Delta^{\mathrm{I}} w(x)-\left(\frac{H_{23}}{H_{22}}\right) \Delta^{\mathrm{I}} \psi(x) \\
& +\left\{\frac{D_{s}}{\pi H_{22}}\right\}[(x-a) \omega(x, a, d) \\
& +(x+a) \omega(-x, a, d)] .
\end{aligned}
$$

4.1.2. Energy Release Rate (ERR). The total energy release rate, $J_{a}$, at the crack tip, $x=a$, is calculated using following crack closure integral:

$$
\begin{aligned}
J_{a}=\frac{1}{2} \lim _{\delta \rightarrow 0} \frac{1}{\delta} \int_{0}^{\delta}[ & \tau_{y z}(r+a, 0) \Delta w(r+a-\delta, 0) \\
& +D_{y}(r+a, 0) \Delta \varphi(r+a-\delta, 0) \\
& \left.+B_{y}(r+a, 0) \Delta \psi(r+a-\delta, 0)\right] d r .
\end{aligned}
$$

Consider

$$
\begin{aligned}
J_{a}= & {\left[\left(\frac{\tau_{s}}{\pi}\right)-\left\{\frac{\left(H_{21} D_{s}\right)}{\left(H_{22} \pi\right)}\right\}\right] } \\
& \times\left[2 a\left(G_{11} \tau_{s}+G_{12} D_{s}\right) \cosh ^{-1}\left(\frac{b}{a}\right)\right. \\
& \left.+\left\{\frac{\left(2 a D_{s}^{2}\right)}{\left(\pi H_{22}\right)}\right\} \cosh ^{-1}\left(\frac{d}{a}\right)-\left\{\frac{\left(B_{23} D_{s}\right)}{\left(2 c H_{22} \pi\right)}\right\} \sqrt{b^{2}-a^{2}}+\left\{\frac{\left(B_{s} G_{13} b^{2}\right)}{2 c}\right\}\right] \\
\times & {\left[4 c b\left(\tau_{\infty}-\tau_{s}\right) G_{31} \cosh ^{-1}\left(\frac{c}{b}\right)\right.} \\
& +\left(G_{33} B_{s} c^{2} \pi\right) \cos ^{-1}\left(\frac{a}{c}\right)+4 a c\left(G_{31} \tau_{s}+G_{32} D_{s}\right) \\
& \left.\times \cosh ^{-1}\left(\frac{c}{a}\right)-\left(4 a B_{s} G_{33} \pi\right) \sqrt{c^{2}-a^{2}}\right] .
\end{aligned}
$$

4.2. Case Study. Piezoelectromagnetic ceramic $\mathrm{BaTiO}_{3}-$ $\mathrm{CoFe}_{2} \mathrm{O}_{4}$ is selected for case study considering $\mathrm{BaTiO}_{3}$ as inclusion and $\mathrm{CoFe}_{2} \mathrm{O}_{4}$ as matrix. The volume fraction of the inclusions is denoted by $V_{i}$. The following mixture rule is used to determine composite material constants, taken from Sih and Song [3]:

$$
\Gamma^{c}=\Gamma^{i} V_{i}+\left(1-V_{i}\right) \Gamma^{m},
$$

where the superscripts $c, i$, and $m$ represent composite, inclusion, and matrix, respectively.

Materials constants for $\mathrm{BaTiO}_{3}-\mathrm{CoFe}_{2} \mathrm{O}_{4}$ for different volume fraction $V_{i}$ are given in Table 1, taken from Sih and Song [4].

The loads fixed for this study are $\tau_{\infty} / \tau_{s}=0.1, D_{\infty} / D_{s}=$ $0.5, B_{\infty} / B_{s}=0.35, D_{\infty} / \tau_{\infty}=3 \times 10^{-10}$ and $B_{\infty} / \tau_{\infty}=0.1 \times$ $10^{-6}$.

As may be noted from Figure 2, CSD shows a linear increase as slide-yield zone length is increased for fixed crack length. But as volume fraction is increased the crack opening decreases in magnitude although the increasing trend is maintained.

Crack opening induction drop (COI) versus induction zone to half-crack length ratio is plotted in Figure 3. COI 
TABLE 1: Material constants of $\mathrm{BaTiO}_{3}-\mathrm{CoFe}_{2} \mathrm{O}_{4}$ for different volume fraction of $\mathrm{BaTiO}_{3}$ in composite material.

\begin{tabular}{lccccc}
\hline Properties & \multicolumn{3}{c}{ Volume fraction of composite } \\
\hline$c_{44}\left(10^{9} \mathrm{~N} / \mathrm{m}^{2}\right)$ & 0.1 & 0.3 & 0.5 & 0.7 & 43.7 \\
$e_{15}\left(\mathrm{C} / \mathrm{m}^{2}\right)$ & 45 & 44.6 & 44 & 8.12 & 43.2 \\
$h_{15}(\mathrm{~N} / \mathrm{Am})$ & 1.16 & 3.48 & 5.8 & 165 & 70.44 \\
$\varepsilon_{11}\left(10^{-10} \mathrm{C}^{2} / \mathrm{Nm}^{2}\right)$ & 495 & 385 & 275 & 55 \\
$d_{11}$ & 11.9 & 34.2 & 56.4 & 0 & 0.6 \\
$\gamma_{11}\left(10^{-6} \mathrm{Ns}^{2} / \mathrm{C}^{2}\right)$ & 0 & 0 & 297 & 100.9 \\
\hline
\end{tabular}

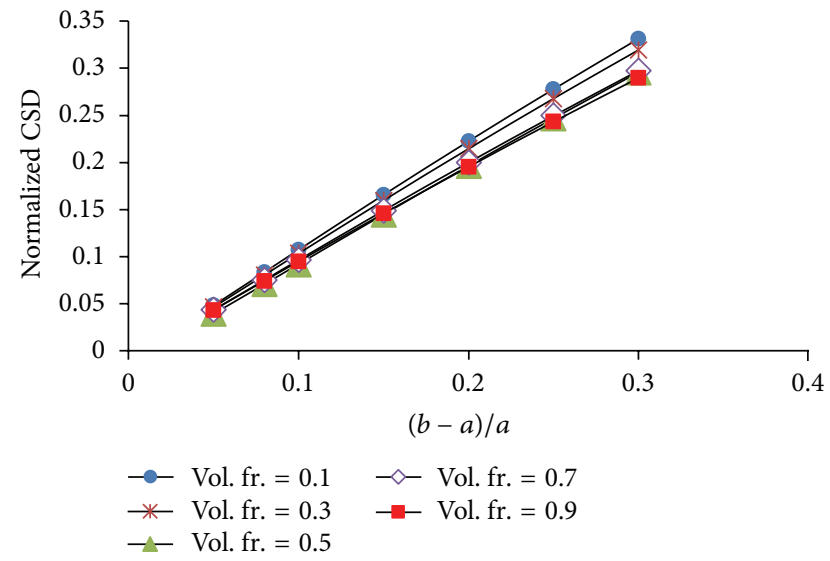

FIGURE 2: Normalized crack sliding displacement versus slide-yield zone to half-crack length ratio for Case I.

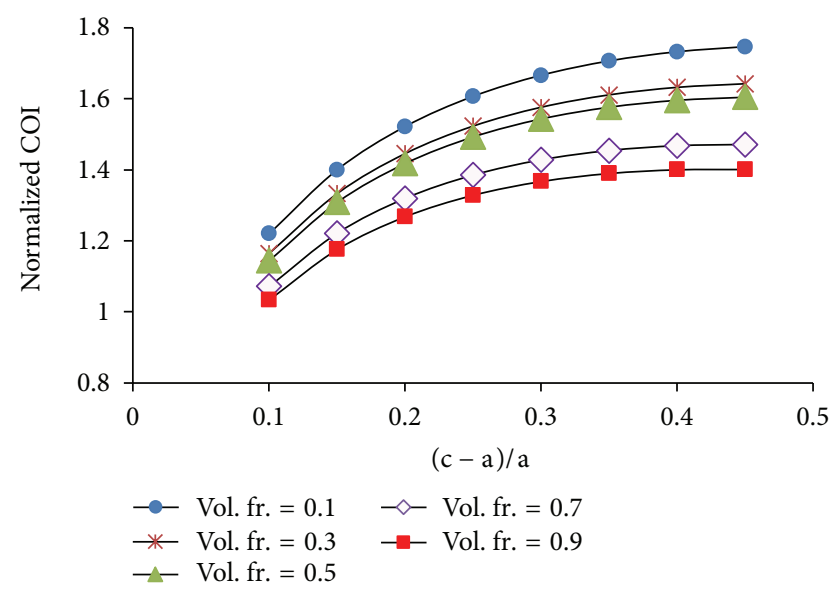

FIGURE 3: Normalized crack opening induction drop versus induction zone to half-crack length ratio for Case I.

shows a nonlinear parabolic increase as induction zone length is increased. COI stabilizes for bigger induction zone. However as the volume fraction is increased, the COI further drops although the increasing trend continues to stabilize.

Figure 4 depicts the variation of crack opening potential drop (COP) with respect to saturation zone length for a fixed crack length. COP shows a negative variation as the saturation zone length is increased. It may be noted that for higher

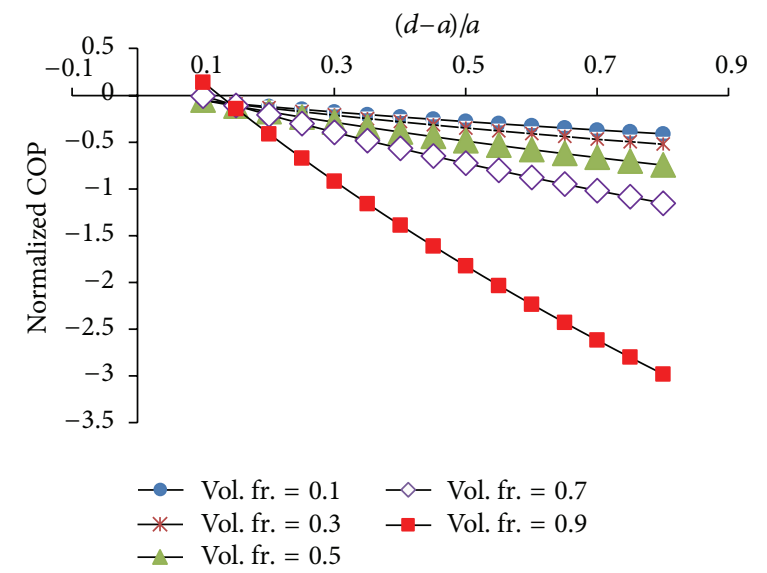

FIGURE 4: Normalized crack opening potential drop versus saturation zone to half-crack length ratio for Case I.

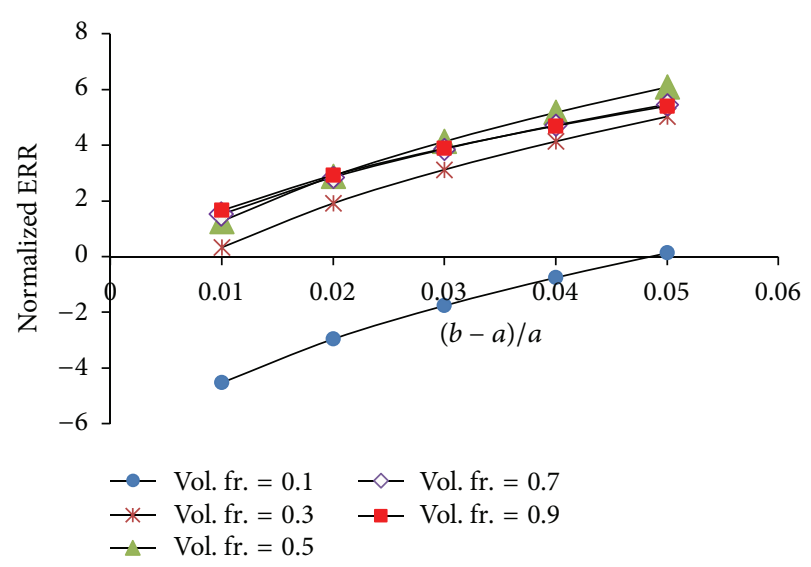

FIGURE 5: Normalized energy release rate versus slide-yield zone to half-crack length ratio for Case I.

volume fraction, there is substantial drop in COP. As the saturation zone length is increased, COP shows a continuous negative increase.

Figure 5 depicts the variation of energy release rate (ERR) with respect to slide-yield zone for different volume fractions. For higher volume fraction, energy release rate is positive and increases as slide-yield zone length is increased. And for $V_{i}>$ 0.7, it is observed that there is little change in ERR variation; 


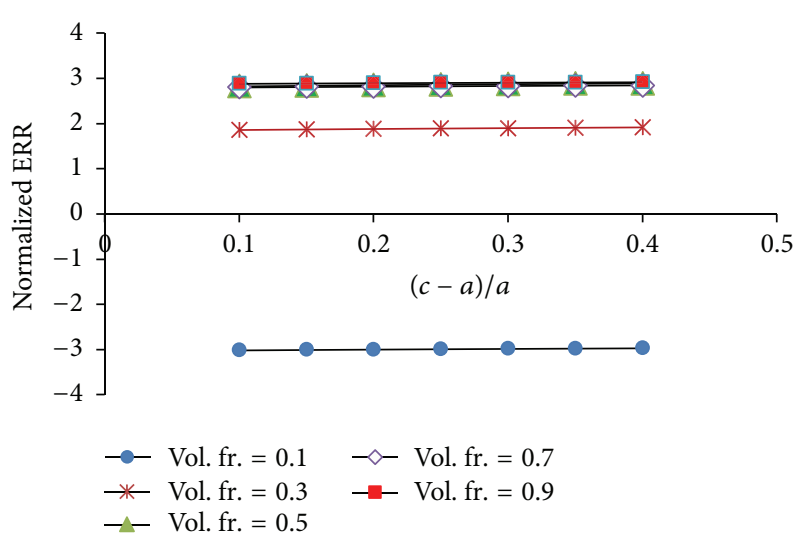

FIGURE 6: Normalized energy release rate versus induction zone to half-crack length ratio for Case I.

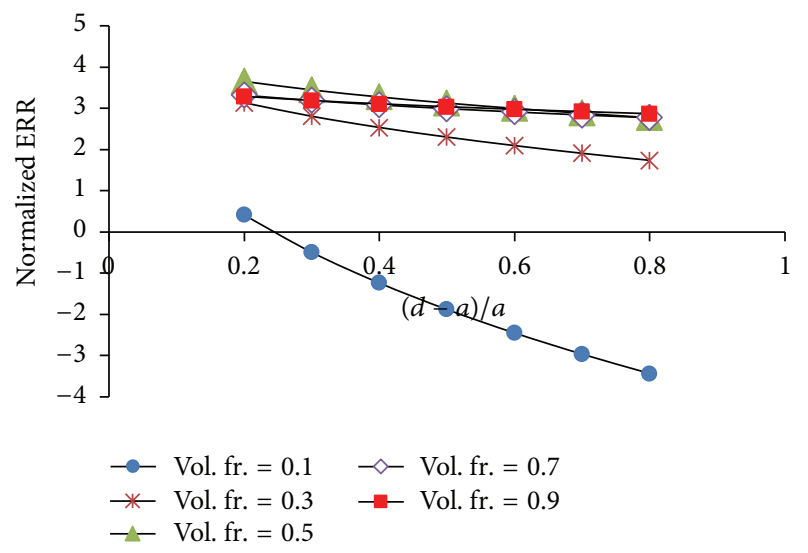

FIGURE 7: Normalized energy release rate versus saturation zone to half-crack length ratio for Case I.

in fact, it slightly decreases for bigger values of slide-yield zone length keeping crack length fixed. It may be noted that, for smaller value of volume fraction, the ERR is negative but increases continuously as slide-yield zone size is increased.

Figure 6 shows the variation of ERR with respect to increase in saturation zone size for different volume fractions. The ERR shows a uniform constant behaviour even when saturation zone is increased. For volume fraction value equal to 0.1 the ERR is negative; it makes no contribution to crack propagation. And for volume fraction value higher than 0.3 , the ERR becomes positive. For volume fraction values equal and higher than 0.5 the ERR remains constant; that is, there is no effect of volume fraction increase.

Variation of ERR against induction zone for different values of volume fraction is plotted in Figure 7. For volume fraction equal to 0.1 the ERR continuously decreases parabolically and becomes negative as the induction zone length is increased. And for the values $0.5-0.9$ of volume fraction the ERR decreases even when induction zone size is increased, indicating that the crack gets arrested for higher volume fraction values.

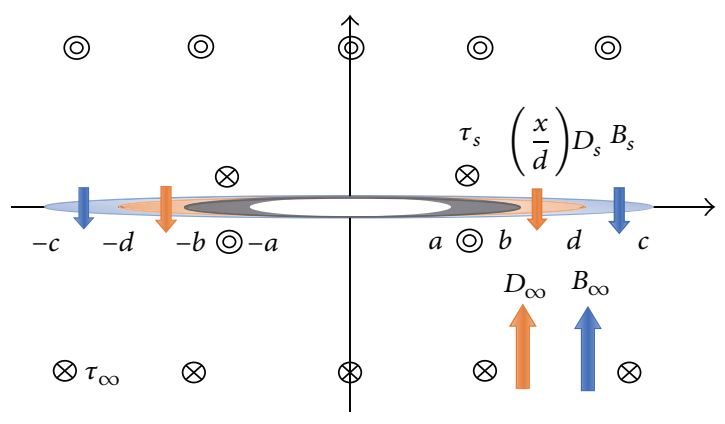

FIGURE 8: The schematic representation of the problem for Case II.

\section{Case II: Solution and Applications}

Schematically, the configuration of the problem is depicted in Figure 8.

The traction boundary conditions $t_{i}^{\mathrm{II}}(i=1,2,3)$ for Case II may be mathematically expressed as

$$
\begin{aligned}
& \text { (iv) } t_{1}^{\mathrm{II}}= \begin{cases}-\tau_{\infty}, & |x|<a, \\
-\tau_{\infty}+\tau_{s}, & a<|x| \leq b,\end{cases} \\
& \text { (v) } t_{2}^{\mathrm{II}}= \begin{cases}-D_{\infty}, & |x| \leq a, \\
-D_{\infty}+\left(\frac{x}{d}\right) D_{s}, & a<|x| \leq d,\end{cases} \\
& \text { (vi) } t_{3}^{\mathrm{II}}= \begin{cases}-B_{\infty}, & |x| \leq a, \\
-B_{\infty}+B_{s}, & a<|x| \leq c,\end{cases}
\end{aligned}
$$

and for this case it is assumed that $a<b<d<c$.

The dislocation function, $g_{1}(x)$, dipole density function, $g_{2}(x)$ and induction density function, $g_{3}(x)$, for this case are denoted by vector $\mathbf{f}(\mathbf{x})$ as

$$
\mathbf{f}(\mathbf{x})=\left[g_{1}(x), g_{2}(x), g_{3}(x)\right]^{T} .
$$

The boundary conditions (iv) to (vi) together with continuity of traction condition on $x$-axis lead to $g_{1}(x)=0$ for $|x|>b$, $g_{2}(x)=0$ for $|x|>d$, and $g_{3}(x)=0$ for $|x|>c$.

Equations to determine the desired potentials $g_{i}(x)(i=$ $1,2,3)$ may be written using (16) as

$$
\begin{aligned}
& \int_{-b}^{b} \frac{g_{1}(t)}{t-x} d t=\pi G_{1 j} t_{j}^{\mathrm{II}}(x), \\
& \int_{-d}^{d} \frac{g_{2}(t)}{t-x} d t=\pi G_{2 j} t_{j}^{\mathrm{II}}(x), \\
& \int_{-c}^{c} \frac{H_{3 j} g_{j}(t)}{t-x} d t=\pi t_{3}^{\mathrm{II}}(x) .
\end{aligned}
$$

The dislocation function, $g_{1}(x)$, from (38) is written as

$$
\begin{aligned}
\pi g_{1}(x)= & \left(G_{11} \tau_{s}+\left(\frac{x}{d}\right) G_{12} D_{s}+G_{13} B_{s}\right) \\
& \times[\omega(x, a, b)-\omega(-x, a, b)] \\
& +\left\{\frac{\left(2 D_{s} G_{12}\right)}{d}\right\} \sqrt{b^{2}-x^{2}} \cos ^{-1}\left(\frac{a}{b}\right),
\end{aligned}
$$


provided that

$$
\begin{gathered}
2\left(G_{11} \tau_{s}+G_{13} B_{s}\right) \cos ^{-1}\left(\frac{a}{b}\right)+\left(\frac{\left(2 D_{s} G_{12}\right)}{d}\right) \sqrt{b^{2}-a^{2}} \\
=\pi\left(G_{11} \tau_{\infty}+G_{12} D_{\infty}+G_{13} B_{\infty}\right) .
\end{gathered}
$$

We can obtained the value of $b$ from (42); then slide-yield zone length is determined using $|b-a|$.

Similarly, dipole density function, $g_{2}(x)$, is obtained, solving (39), as

$$
\begin{aligned}
\pi g_{2}(x)= & G_{21}\left(\tau_{\infty}-\tau_{s}\right)[\omega(x, b, d)-\omega(-x, b, d)] \\
& +2 D_{s} G_{22} \sqrt{1-\left(\frac{x}{d}\right)^{2} \cos ^{-1}\left(\frac{a}{d}\right)} \\
& +\left\{G_{21} \tau_{s}+\left(\frac{x}{d}\right) G_{22} D_{s}+G_{23} B_{s}\right\} \\
& \times[\omega(x, a, d)-\omega(-x, a, d)]
\end{aligned}
$$

under the condition

$$
\begin{aligned}
& G_{21}\left(\tau_{\infty}-\tau_{s}\right) \cos ^{-1}\left(\frac{b}{d}\right) \\
& \quad+D_{s} G_{22} \sqrt{1-\left(\frac{a}{d}\right)^{2}}+\left(G_{23} B_{s}+G_{21} \tau_{s}\right) \cos ^{-1}\left(\frac{a}{d}\right) \\
& =\left(\frac{\pi}{2}\right)\left(G_{21} \tau_{\infty}+G_{22} D_{\infty}+G_{23} B_{\infty}\right) .
\end{aligned}
$$

This condition enables to determine $d$. The saturation zone is obtained from $|d-a|$.

Having determined $g_{1}(x)$ and $g_{2}(x)$ the induction function $g_{3}(x)$ is now determined from (40) as

$$
\begin{aligned}
H_{33} g_{3}(x)= & -H_{31} g_{1}(x)-H_{32} g_{2}(x) \\
& +\left(\frac{B_{s}}{\pi}\right)[\omega(x, a, c)-\omega(-x, a, c)],
\end{aligned}
$$

under the constrain

$$
\frac{c}{a}=\sec \left(\frac{\pi}{2} \frac{B_{\infty}}{B_{s}}\right)
$$

The above equation enables to determine $c$, hence giving induction zone length using $|c-a|$.

\subsection{Applications for Case II}

5.1.1. CSD, COP, and COI Calculations. The crack sliding displacement, $\Delta^{\mathrm{II}} w(x)$, is calculated using following formula:

$$
\Delta^{\mathrm{II}} w(x)=-\int_{-b}^{x} g_{1}(x) d x, \quad-b \leq x \leq b
$$

Substituting $g_{1}(x)$ from (41) into the above relation and evaluating, one obtains

$$
\begin{aligned}
\Delta^{\mathrm{II}} w(x) & \\
=\left(\frac{1}{\pi}\right)[ & \left(G_{11} \tau_{s}+G_{13} B_{s}\right) \\
& \times[(x-a) \omega(x, a, b)+(x+b) \omega(-x, a, b)] \\
& -\left\{\frac{\left(D_{s} G_{12}\right)}{d}\right\}\left[\left(x^{2}-a^{2}\right)\{\omega(x, a, b)+\omega(-x, a, b)\}\right. \\
& -\left\{\frac{\left(D_{s} G_{12}\right)}{d}\right\} \cos ^{-1}\left(\frac{a}{b}\right) \\
& \left.\times\left[x \sqrt{b^{2}-x^{2}}+b^{2} \sin ^{-1}\left(\frac{x}{b}\right)-\frac{\left(b^{2} \pi\right)}{2}\right]\right] .
\end{aligned}
$$

The crack opening potential drop (COP), $\Delta^{\mathrm{II}} \phi(x)$, is calculated as

$$
\begin{aligned}
\Delta^{\mathrm{II}} \phi(x)= & -\int_{-d}^{x} g_{2}(x) d x, \quad-d \leq x \leq d, \\
= & \left(\frac{1}{\pi}\right) G_{21}\left(\tau_{\infty}-\tau_{s}\right) \\
& \times[(x-b) \omega(x, b, d)+(x+b) \omega(-x, b, d)] \\
& -\left\{\frac{\left(D_{s} G_{22}\right)}{(\pi d)}\right\} \cos ^{-1}\left(\frac{a}{d}\right) \\
& \times\left[x \sqrt{d^{2}-x^{2}}+c^{2} \sin ^{-1}\left(\frac{x}{d}\right)-\frac{\left(d^{2} \pi\right)}{2}\right] \\
& +\left\{\frac{\left(G_{21} \tau_{s}+G_{23} B_{s}\right)}{\pi}\right\} \\
& \times[(x-a) \omega(x, a, d)+(x+a) \omega(-x, a, d)] \\
& \left.-2 a \sqrt{d^{2}-a^{2}}\left(\cos ^{-1}\left(\frac{a}{d}\right)-\pi\right)\right] . \\
& \times\left[\left(x^{2}-a^{2}\right)\{\omega(x, a, d)+\omega(-x, a, d)\}\right.
\end{aligned}
$$

The in-plane crack opening potential drop, $\Delta^{\mathrm{II}} \psi(x)$, is written as

$$
\begin{aligned}
\Delta^{\mathrm{II}} \psi(x) & =-\int_{-c}^{x} g_{3}(x) d x, \quad-c \leq x \leq c, \\
& =-\left(\frac{H_{31}}{H_{33}}\right) \Delta^{\mathrm{II}} w(x)-\left(\frac{H_{32}}{H_{33}}\right) \Delta^{\mathrm{II}} \phi(x)
\end{aligned}
$$




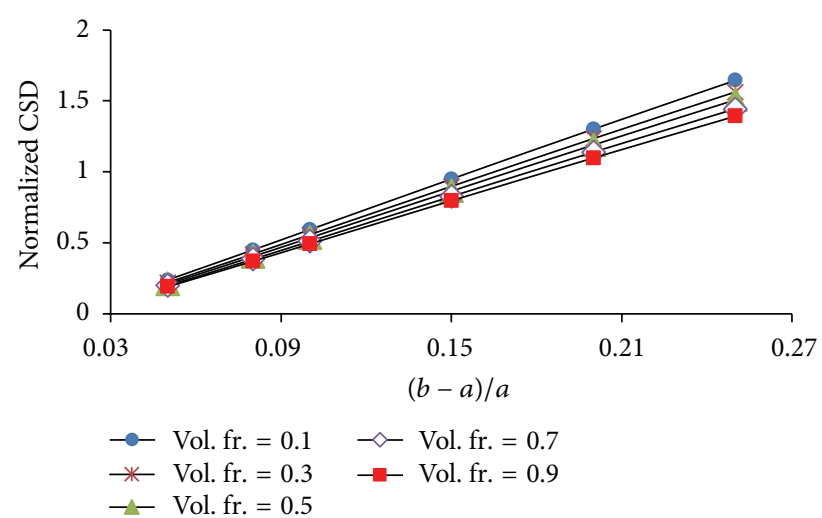

FIGURE 9: Normalized crack sliding displacement versus slide zone to half-crack length ratio for Case II.

$$
\begin{aligned}
& +\left\{\frac{B_{s}}{\left(\pi H_{33}\right)}\right\} \\
& \times[(x-a) \omega(x, a, c)+(x+a) \omega(-x, a, c)] .
\end{aligned}
$$

5.1.2. Energy Release Rate (ERR). Using crack closure integral from (33), the ERR, $J_{a}$, is calculated at the crack tip, $x=a$, for this case as

$$
\begin{aligned}
& J_{a}=\left\{\left(\frac{\tau_{s}}{\pi}\right)-\left(\frac{\left(H_{31} D_{s}\right)}{\left(H_{33} \pi\right)}\right)\right\} \\
& \times\left[2 a\left(G_{11} \tau_{s}+G_{13} B_{s}\right) \cosh ^{-1}\left(\frac{b}{a}\right)\right. \\
& \left.-\left\{\frac{\left(2 D_{s} G_{12}\right)}{d}\right\} \sqrt{b^{2}-a^{2}}+\left(\frac{\left(D_{s} G_{12} b^{2}\right)}{2 d}\right)\right] \\
& +\left(\frac{\left(2 a B_{s}^{2}\right)}{\left(\pi H_{33}\right)}\right) \cosh ^{-1}\left(\frac{c}{a}\right)-\left(\frac{\left(H_{32} B_{s}\right)}{\left(2 d H_{33} \pi\right)}\right) \\
& \times\left[4 d b\left(\tau_{\infty}-\tau_{s}\right) G_{21} \cosh ^{-1}\left(\frac{d}{b}\right)\right. \\
& +\left(G_{22} D_{s} d^{2} \pi\right) \cos ^{-1}\left(\frac{a}{d}\right) \\
& +4 a d\left(G_{21} \tau_{s}+G_{23} B_{s}\right) \\
& \left.\times \cosh ^{-1}\left(\frac{d}{a}\right)-\left(4 a D_{s} G_{22} \pi\right) \sqrt{d^{2}-a^{2}}\right] .
\end{aligned}
$$

5.2. Case Study for Case II. For this case also the composite $\mathrm{BaTiO}_{3}-\mathrm{CoFe}_{2} \mathrm{O}_{4}$ is selected. Consequently, the material constants remain the same as in Case I, given in Table 1.

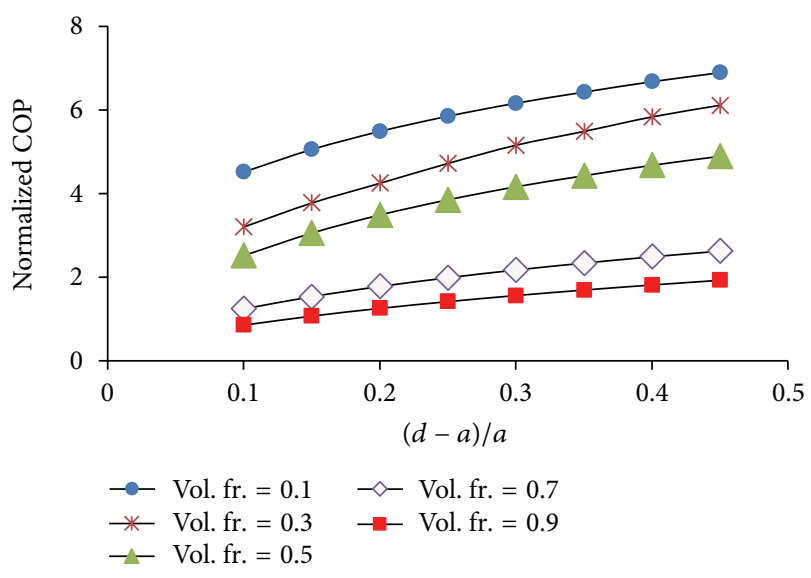

FIGURE 10: Normalized crack opening potential drop versus saturation zone to half-crack length ratio for Case II.

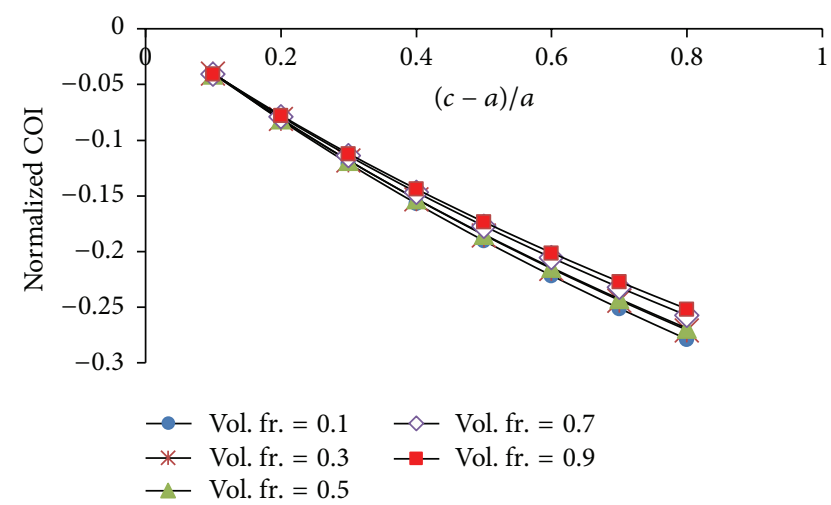

FIGURE 11: Normalized crack opening induction drop versus induction zone to half-crack length ratio for Case II.

The load ratios prescribed for this case are $\tau_{\infty} / \tau_{s}=0.17$, $D_{\infty} / D_{s}=0.5, B_{\infty} / B_{s}=0.7, D_{\infty} / \tau_{\infty}=3 \times 10^{-10}$, and $B_{\infty} / \tau_{\infty}=0.1 \times 10^{-6}$.

Figure 9 depicts variation of normalized crack sliding displacement versus slide-yield zone length, for fixed crack length. It is observed that as slide-yield zone length is increased then crack opens more. It is interesting to note that for higher values of volume fraction, crack opens less. It is to be noted that CSD for Case II is almost three times higher than that for Case I.

Variation of COP vis-à-vis saturation zone length (for fixed crack length) is plotted in Figure 10. It is observed that $\mathrm{COP}$ increases and remains positive as saturation zone length is increased. The behavior is in contrast with that in Case I where COP throughout remains negative for all volume fractions. It is also to be noted that for higher volume fraction values (>0.5) COP also drops, continuously.

Figure 11 depicts the variation of COI with respect to induction zone. It is observed that as induction zone size is increased, the COI decreases continuously. The significant point to be noted here is that COI for this case remains 


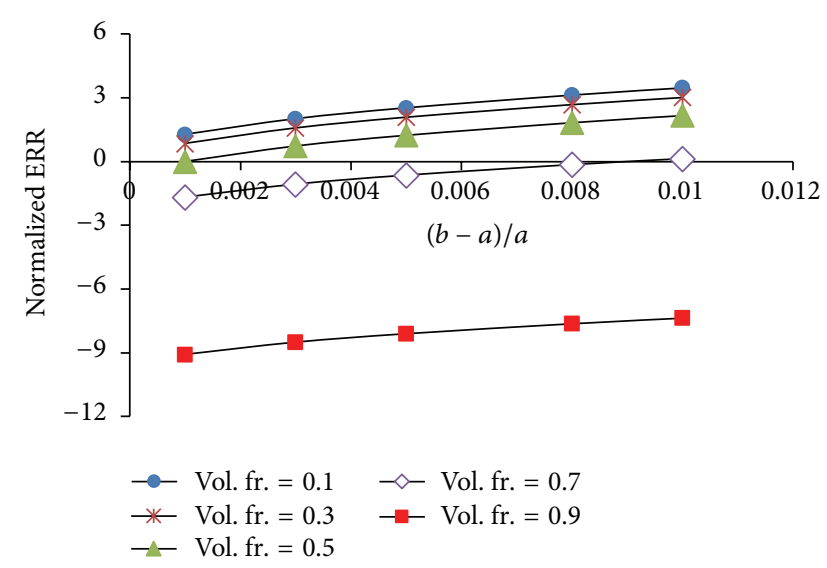

FIGURE 12: Normalized energy release rate versus slide-yield zone to half-crack length ratio for Case II.

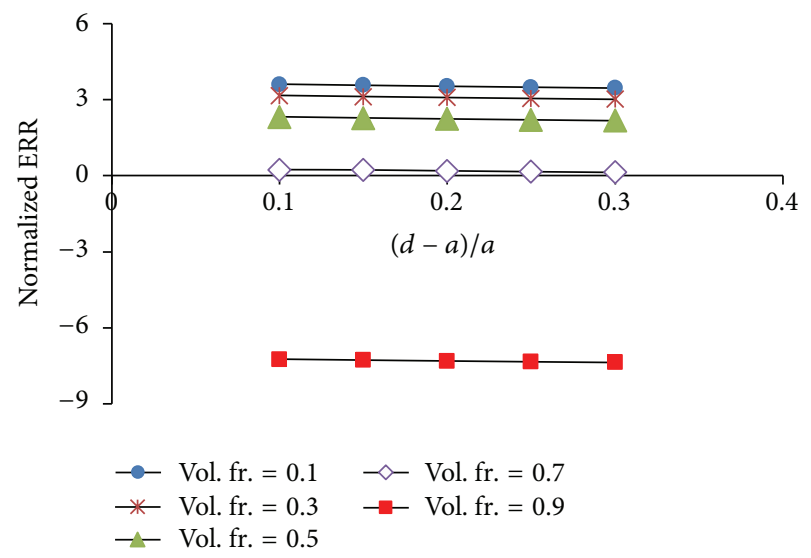

FIGURE 13: Normalized energy release rate versus saturation zone to half-crack length ratio for Case II.

negative which is in contrast to Case I where it was positive. This is due to the fact that the two zones have interchanged their rolls. The COI shows a little variation as volume fraction is increased.

Variation in ERR with respect to slide-yield zone increase is drawn in Figure 12. It is observed that as the length of slide-yield zone increases the ERR increases too, as expected. The important fact to be noted is that with volume fraction increase, the energy release rate drops and becomes negative for higher values of the volume fraction. Also their behavior is in contrast to that in Case I where ERR was increasing for increasing values of volume fraction.

Figure 13 captures the variation of ERR with respect to induction zone. It is noted that ERR is uniformly constant. It is to be noted that as the value of volume fraction increases ERR reduces and becomes negative for higher values of volume fraction. This implies that the energy is generated but not utilized in crack opening.

Figure 14 shows that ERR reduce as the saturation zone is increased confirming crack arrest situation. Also ERR reduces and becomes negative as volume fraction is increased.

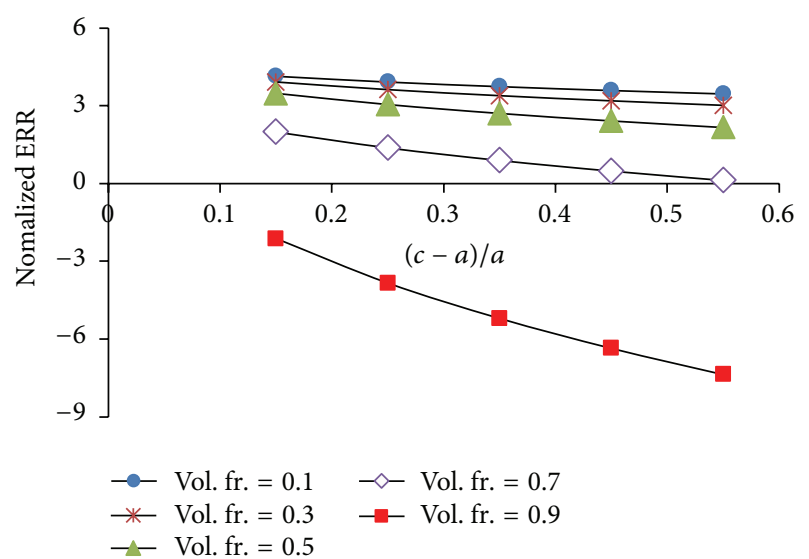

FIGURE 14: Normalized energy release rate versus induction zone to half-crack length ratio for Case II.

This also affirms that the crack opening gets arrested as volume fraction is increased. It is to be noted that for higher value of volume fraction (bigger than 0.7 ) the energy released does not assist in crack opening.

\section{Conclusions}

(i) A strip-yield-saturation-induction model is proposed for a cracked unbounded piezoelectromagnetic media under antiplane shear stress and in-plane electric and magnetic loading conditions.

(ii) Closed form analytic expressions are obtained for crack sliding displacement, crack opening potential drop, crack opening induction drop, developed slide-yield zone, saturation and induction zones, and energy release rate.

(iii) Solutions are obtained for two cases considered, Case I is when developed saturation zone exceeds the developed induction zone and Case II is when saturation zone is smaller than the induction zone. In both cases the developed slide-yield zone is the smallest.

(iv) The case study is presented for $\mathrm{BaTiO}_{3}-\mathrm{CoFe}_{2} \mathrm{O}_{4}$ composite, for both cases.

(v) COP and COI for both Cases I and II reverse their rolls. In Case I, COI remains positive while COP remains negative and in Case II, COP remains positive and COI becomes negative, for all values of volume fraction.

(vi) The negative energy release rate variation for higher values of volume fraction confirms that it does not assist in crack opening. And ERR's decreasing variation with respect to saturation zone increase also concludes the possible arrest of crack opening.

\section{Conflict of Interests}

The authors declare that there is no conflict of interests regarding the publication of this paper. 


\section{Acknowledgments}

The authors are grateful to Professor R. D. Bhargava\{Senior Professor and Head (retired), Indian Institute of Technology Bombay, Mumbai\} for continuous encouragement during the course of this work. The second author is thankful to MHRD for the financial support.

\section{References}

[1] X.-M. Wang and Y.-P. Shen, "The conservation laws and pathindependent integrals with an application for linear electromagneto-elastic media," International Journal of Solids and Structures, vol. 33, no. 6, pp. 865-878, 1996.

[2] L. Jinxi, L. Xianglin, and Z. Yongbin, “Green's functions for anisotropic magnetoelectroelastic solids with an elliptical cavity or a crack," International Journal of Engineering Science, vol. 39, no. 12, pp. 1405-1418, 2001.

[3] G. C. Sih and Z. F. Song, "Magnetic and electric poling effects associated with crack growth in $\mathrm{BaTiO}_{3}-\mathrm{CoFe}_{2} \mathrm{O}_{4}$ composite," Theoretical and Applied Fracture Mechanics, vol. 39, no. 3, pp. 209-227, 2003.

[4] Z. F. Song and G. C. Sih, "Crack initiation behavior in magnetoelectroelastic composite under in-plane deformation," Theoretical and Applied Fracture Mechanics, vol. 39, no. 3, pp. 189-207, 2003.

[5] B. L. Wang and Y. Mai, "Crack tip field in piezoelectric/piezomagnetic media," European Journal of Mechanics A/Solids, vol. 22, no. 4, pp. 591-602, 2003.

[6] B. L. Wang and Y. W. Mai, "Fracture of piezo-electro-magnetic materials," Mechanics Research Communications, vol. 35, pp. 6573, 2004.

[7] C. Gao, P. Tong, and T. Zhang, "Fracture mechanics for a mode III crack in a magnetoelectroelastic solid," International Journal of Solids and Structures, vol. 41, no. 24-25, pp. 6613-6629, 2004.

[8] K. Hu and G. Li, "Electro-magneto-elastic analysis of a piezoelectromagnetic strip with a finite crack under longitudinal shear," Mechanics of Materials, vol. 37, no. 9, pp. 925-934, 2005.

[9] W. Y. Tian and R. K. N. D. Rajapakse, "Fracture analysis of magnetoelectroelastic solids by using path independent integrals," International Journal of Fracture, vol. 131, no. 4, pp. 311-335, 2005.

[10] M. H. Zhao, H. Wang, F. Yang, and T. Liu, "A magnetoelectroelastic medium with an elliptical cavity under combined mechanical-electric-magnetic loading," Theoretical and Applied Fracture Mechanics, vol. 45, no. 3, pp. 227-237, 2006.

[11] B. Wang and Y. Mai, "Applicability of the crack-face electromagnetic boundary conditions for fracture of magnetoelectroelastic materials," International Journal of Solids and Structures, vol. 44, no. 2, pp. 387-398, 2007.

[12] L. Ma, J. Li, R. Abdelmoula, and L. Wu, "Mode III crack problem in a functionally graded magneto-electro-elastic strip," International Journal of Solids and Structures, vol. 44, no. 17, pp. 55185537, 2007.

[13] X. Zhong and X. Li, "Magnetoelectroelastic analysis for an opening crack in a piezoelectromagnetic solid," European Journal of Mechanics, A/Solids, vol. 26, no. 3, pp. 405-417, 2007.

[14] V. S. Kirilyuk, "Stress state of a transversely isotropic magnetoelectroelastic body with a plane crack under antisymmetric loads," International Applied Mechanics, vol. 44, no. 10, pp. 11061118, 2008.
[15] M. Zhao and C. Fan, "Strip electric-magnetic breakdown model in a magnetoelectroelastic medium," Journal of the Mechanics and Physics of Solids, vol. 56, no. 12, pp. 3441-3458, 2008.

[16] C. Y. Fan and M. H. Zhao, "Nonlinear fracture of 2D magnetoelectroelastic media: analytical and numerical solutions," International Journal of Solids and Structures, vol. 48, no. 16-17, pp. 2383-2392, 2011.

[17] S. Krahulec, J. Sladek, V. Sladek, and P. Stanak, "Crack analysis in magnetoelectroelastic solids," in Proceedings of the 18th International Conference Engineering Mechanics, Paper no. 56, pp. 709718, Svratka, Czech Republic, May 2012.

[18] R. R. Bhargava and P. Raj Verma, "A crack arrest study for piezoelectro-magnetic media under mechanical, electric and magnetic small-scale-yielding," Communications in Computer and Information Science, vol. 283, no. 1, pp. 374-383, 2012.

[19] D. S. Dugdale, "Yielding of steel sheets containing slits," Journal of the Mechanics and Physics of Solids, vol. 8, no. 2, pp. 100-104, 1960. 


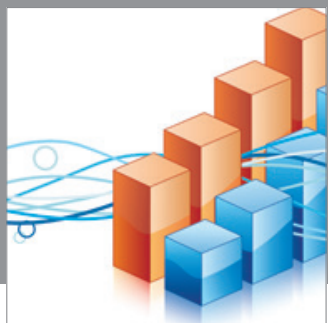

Advances in

Operations Research

mansans

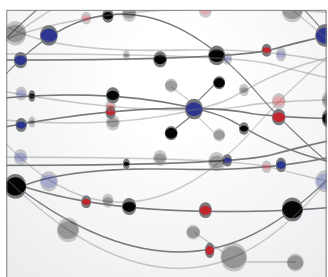

The Scientific World Journal
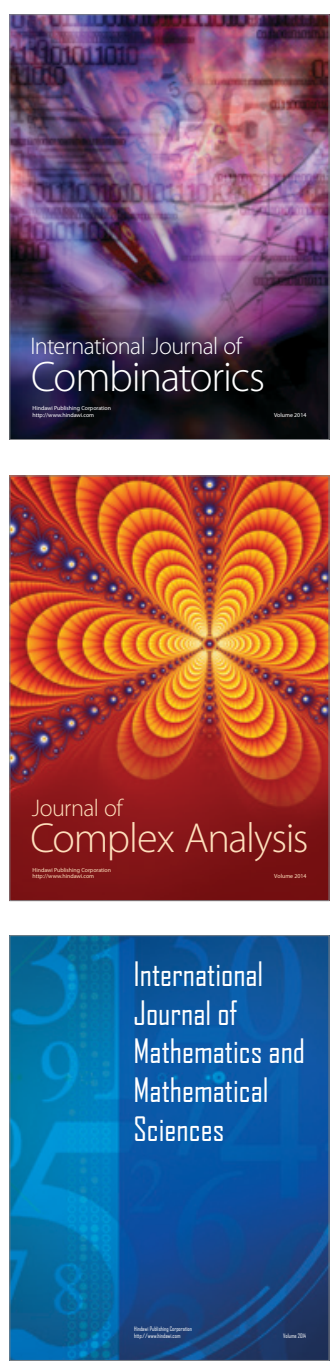
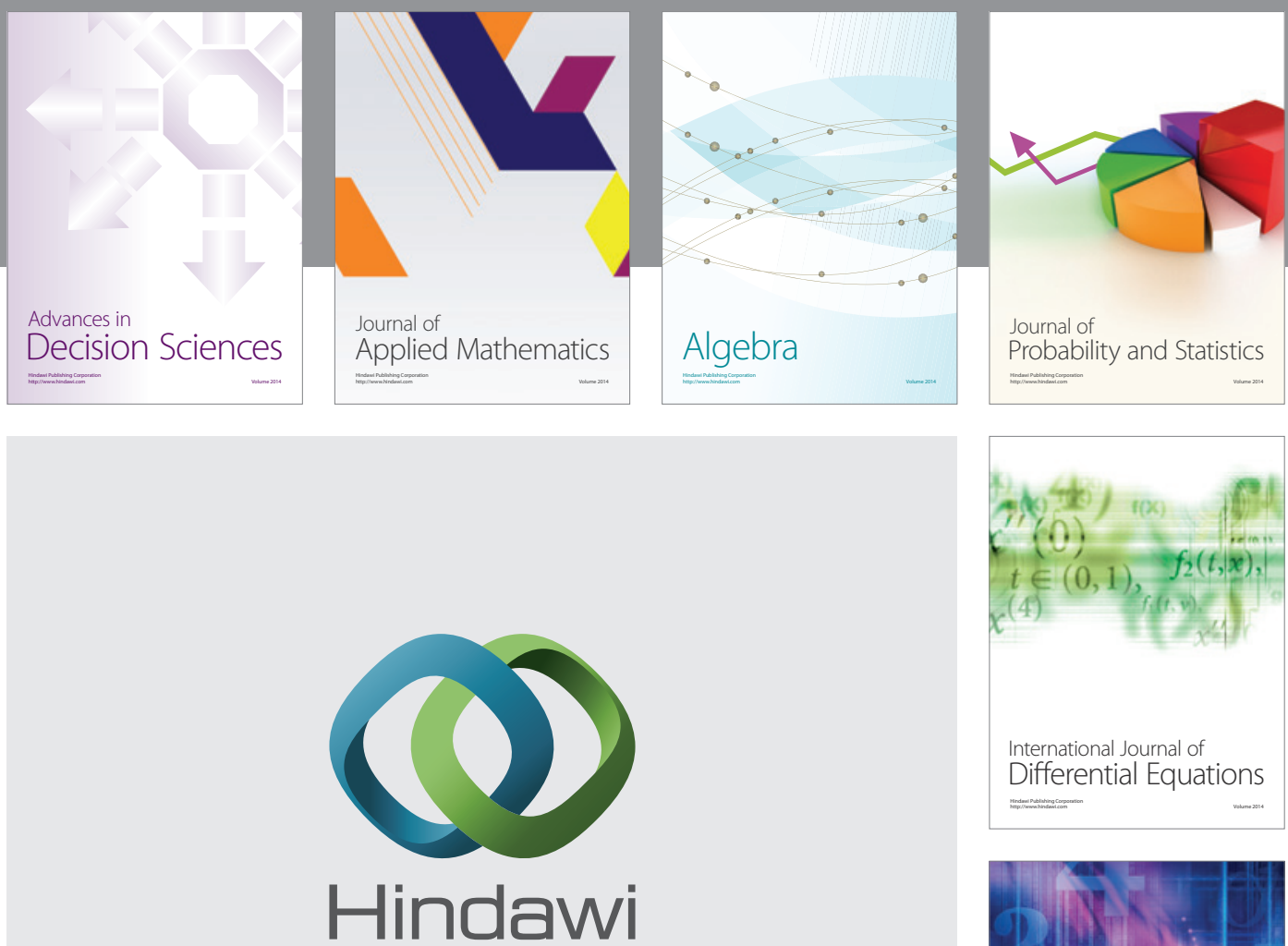

Submit your manuscripts at http://www.hindawi.com
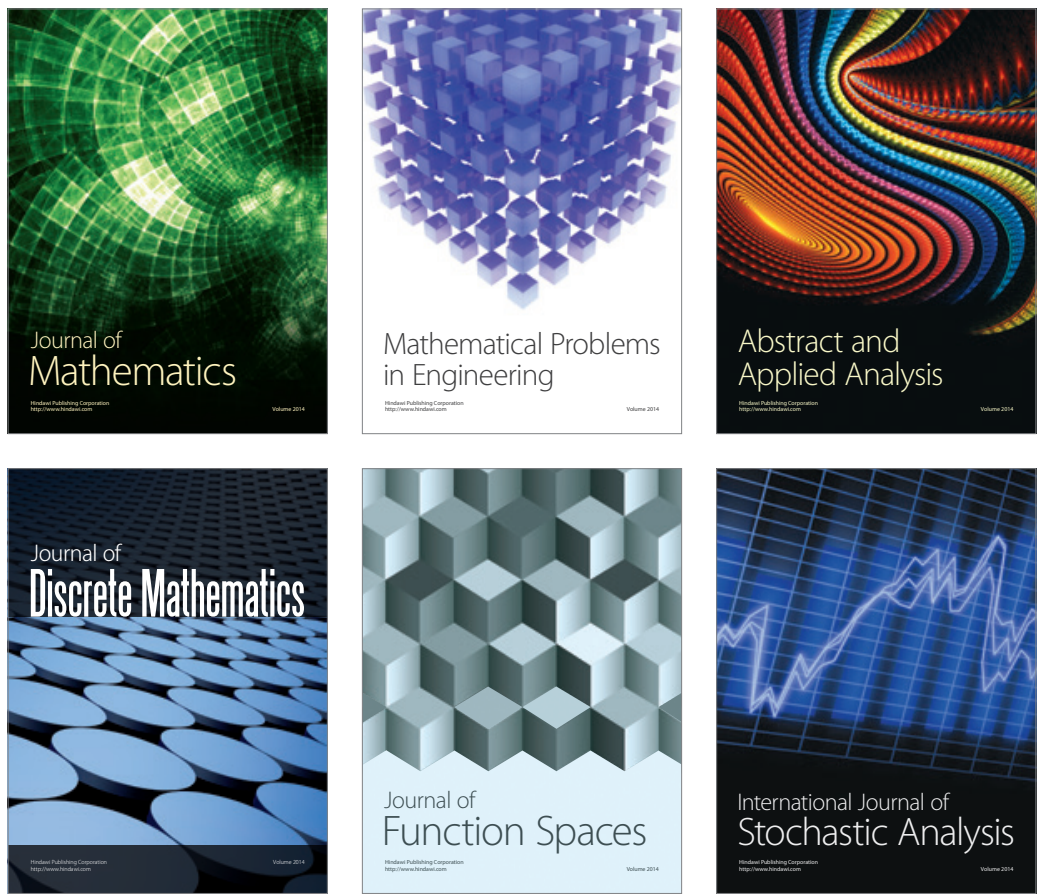

Journal of

Function Spaces

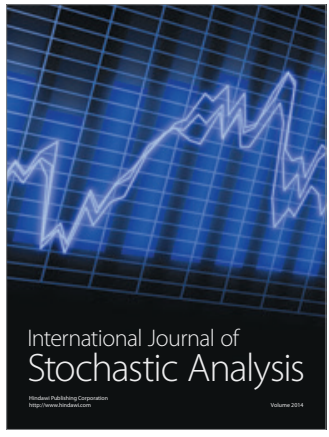

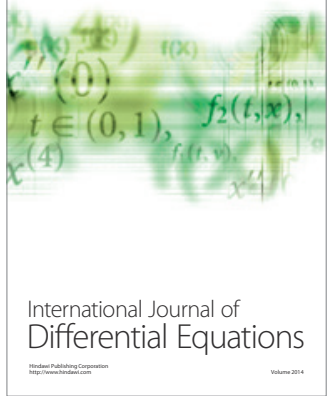
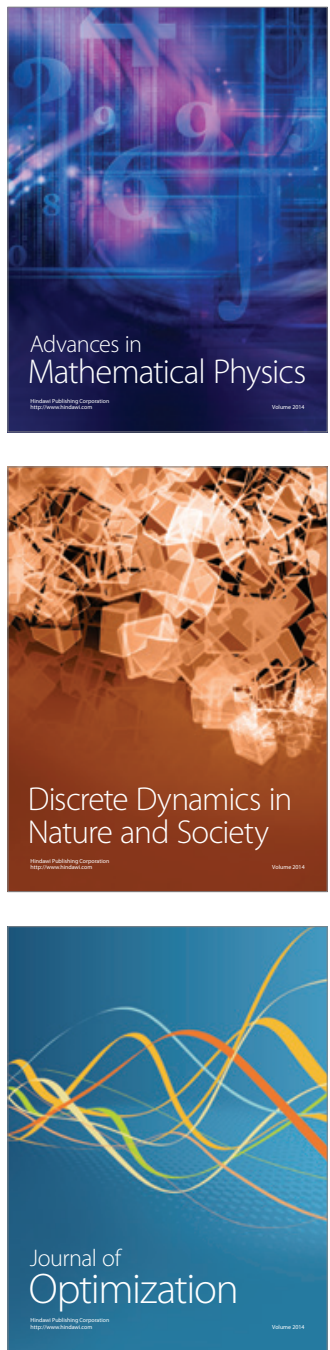